\title{
Kant y la teleología de la naturaleza: acerca de la intención de la naturaleza en Idea para una historia universal en intención cosmopolita y la garantía de la naturaleza en Hacia la paz perpetua ${ }^{*}$
}

\author{
Kant and the teleology of nature: on the aim of nature in Idea for $a$ \\ universal history with a cosmopolitan aim and the guarantee of nature in \\ Toward perpetual peace
}

Por: Arias Albisu, Martín *

CONICET

Argentina

Email: arias.martin@gmail.com

Código Orcid: 0000-0001-9835-4816

Fecha de recepción: 14/10/2021

Fecha de aprobación: 29/11/2021

DOI: http://dx.doi.org/10.30972/nvt.1725717

\section{Resumen}

En Idea para una historia universal en intención cosmopolita (1784), Kant presenta el concepto de una intención de la naturaleza que confiere sentido y finalidad a la historia de la humanidad. El concepto de esta intención es una idea regulativa de la razón teórica, y está fundamentado entonces por la doctrina de estas ideas presentada por Kant en su Crítica de la razón pura. La finalidad de la naturaleza para el ser humano es el desarrollo completo de las disposiciones humanas, especialmente la racional. Como estas disposiciones se desarrollan máximamente en el marco de una constitución civil justa, y como el establecimiento de esta constitución sería inútil si hubiese una situación de guerra entre los diferentes Estados, se torna necesario

\footnotetext{
* Este texto se inscribe en el marco de mi investigación actual en Conicet (período 2021-2022): "Acerca de las ideas rectoras de las concepciones kantianas de la historia y la antropología pragmática".

* Doctor en Humanidades y Artes con mención en Filosofía por la Universidad Nacional de Rosario (Argentina). Realizó estudios de posdoctorado en Alemania y Bélgica. Se desempeña actualmente como Investigador Adjunto del CONICET. Publicó 25 artículos en revistas arbitradas y participó en numerosos congresos nacionales e internacionales.
} 
Kant y la teleología de la naturaleza: acerca de la intención de la naturaleza en Idea para una historia universal en intención cosmopolita y la garantía de la naturaleza en Hacia la paz perpetua

instituir una confederación de los mismos, poseedora de un poder coactivo, para dirimir legalmente los conflictos. En Hacia la paz perpetua (1795), en cambio, Kant presenta el concepto de una garantía de la naturaleza para la aproximación asintótica del ser humano hacia una condición de paz perpetua. La forma política de esta condición es la de una confederación voluntaria de Estados sin constitución ni poder coactivo. La mencionada garantía de la naturaleza no es de índole teórica, sino práctica, porque su función no consiste en proporcionar conocimiento, sino en sustentar la finalidad moral de realizar asintóticamente la idea de la paz perpetua. Esta garantía de la naturaleza es fundamentada por la doctrina teleológica de la naturaleza expuesta en la Crítica de la facultad de juzgar. El objetivo de este artículo es presentar detalladamente las diferencias y semejanzas entre las dos concepciones teleológicas de la naturaleza que acabamos de esbozar.

Palabras clave: Kant - Teleología - Naturaleza

\section{Abstract}

In Idea for a universal history in cosmopolitan intention (1784), Kant presents the concept of an intention of nature that gives meaning and purpose to the history of humanity. The concept of this intention is a regulative idea of theoretical reason, and is then grounded by the doctrine of these ideas presented by Kant in his Critique of Pure Reason. The purpose of nature for the human being is the complete development of human dispositions, especially the rational one. As these dispositions are developed maximally within the framework of a just civil constitution, and as the establishment of this constitution would be useless if there were a situation of war between the different States, it becomes necessary to institute a confederation of them, possessing a coercive power, to legally settle conflicts. In Toward perpetual peace (1795), on the other hand, Kant presents the concept of a guarantee of nature for the asymptotic approach of the human being toward a condition of perpetual peace. The political form of this condition is that of a voluntary confederation of States without constitution or coercive power. The aforementioned guarantee of nature is not of a theoretical character, but of a practical character, because its function is not to provide 


\section{Martín Arias Albisu}

knowledge, but to support the moral purpose of realizing the idea of perpetual peace asymptotically. This guarantee of nature is based on the teleological doctrine of nature set forth in the Critique of the power of judgment. The objective of this article is to present in detail the differences and similarities between the two teleological conceptions of nature that we have just outlined.

Keywords: Kant - Teleology - Nature

\section{Cómo citar este artículo:}

APA: Arias Albisu, M. (2021). Kant y la teleología de la naturaleza: acerca de la intención de la naturaleza en Idea para una historia universal en intención cosmopolita y la garantía de la naturaleza en Hacia la paz perpetua. Nuevo Itinerario, 17 (2), 127158. Recuperado de: (agregar dirección web)

En Idea para una historia universal en intención cosmopolita (laG, 1784), Kant introduce el concepto de una "intención de la naturaleza" [Naturabsicht] que permite reconocer una finalidad en el decurso de la historia humana en su conjunto. En Hacia la paz perpetua (ZeF, 1795), en cambio, se presenta el concepto de una "garantía" [Garantie] de la naturaleza para la consecución de la finalidad de la paz perpetua. El objetivo del presente trabajo es exponer estas dos concepciones y examinar sus semejanzas y diferencias. A fin de conseguir este propósito, examinaremos en la primera sección la argumentación de laG acerca de la intención de la naturaleza. En la segunda sección, estudiaremos la argumentación de ZeF acerca de la garantía de la naturaleza. En la sección siguiente, presentaremos las diferencias principales entre las dos mencionadas argumentaciones. En la sección cuarta, nos ocuparemos del problema de la fundamentación de la teleología de la naturaleza de laG y de ZeF. ${ }^{1}$

\footnotetext{
${ }^{1}$ Ofrecemos las referencias a los textos de Kant según la edición académica de sus obras. Con este fin, empleamos la sigla "AA" (por Akademie-Ausgabe), seguida de los números de tomo y de página (Kant, 1900ss). Las referencias a la Crítica de la razón pura se dan, en conformidad con la usanza habitual, con la letra "A" para la primera edición de 1781, y con la letra "B" para la segunda edición de 1787. Empleamos las abreviaturas estándares de las obras kantianas contenidas en las instrucciones para los autores de la revista Kant Studien. Indicamos la abreviatura de cada obra de Kant al mencionar en nuestro trabajo a esta obra por primera vez. Las abreviaturas pueden consultarse en: http://www.kant-
} 
Kant y la teleología de la naturaleza: acerca de la intención de la naturaleza en Idea para una historia universal en intención cosmopolita y la garantía de la naturaleza en Hacia la paz perpetua

\section{La argumentación acerca de la intención la naturaleza en laG}

Al comienzo de laG, Kant afirma que, si bien las acciones individuales humanas dan la impresión de ser enmarañadas e irregulares, tal vez pueda encontrarse en la especie humana considerada en su conjunto un "desarrollo en constante progreso [...] de las disposiciones originarias de la misma" (laG, AA 08: 17. Traducción modificada). ${ }^{2}$

Como los seres humanos no actúan solamente ni por instinto ni en conformidad con la razón, parecería que no es posible encontrar un plan en la historia de la humanidad. En este punto de la argumentación, a saber, el referido a la posibilidad de encontrar un plan en la historia humana, introduce Kant el concepto de una intención de la naturaleza.

En este orden de cosas, al filósofo no le queda otro recurso -puesto que no puede presuponer en los seres humanos y su actuación global ningún propósito racional propio- que intentar descubrir en este absurdo decurso de las cosas humanas una intención de la Naturaleza, a partir de la cual sea posible una historia de criaturas tales que, sin conducirse con arreglo a un plan propio, sí lo hagan conforme a un determinado plan de la Naturaleza (laG, AA 08: 18. Traducción modificada).

Los individuos no advierten que, al buscar cada uno su propio interés, siguen sin saberlo la mencionada intención de la naturaleza (laG, AA 08: 17). La conclusión que puede extraerse de esta presentación inicial, por parte de Kant, del concepto de una intención de la naturaleza, es que el mismo es de índole teórica, dado que es

gesellschaft.de/de/ks/HinweiseAutorenSiglen neu.pdf. Acerca de las traducciones castellanas empleadas, véase la bibliografía incluida al final de este trabajo.

2 Louden explica que el concepto de disposición [Anlage] hace referencia a "las tendencias hereditarias" de una especie. La "actualización completa" de estas tendencias "depende de factores ambientales favorables". En el caso de las disposiciones de los seres humanos, queda a cargo de estos seres el crear las condiciones para que estas disposiciones se desarrollen adecuadamente (Louden, 2014, pp. 215-216. Véase Pedagogía; Päd, AA 09: 445). Shell, por su parte, aclara que el término "Anlage", al igual que su equivalente latino "dispositio", proviene del campo de la biología. Significa, la mayoría de las veces, capacidades físicas, intelectuales y morales que la naturaleza confiere a un ser (Shell, 2015, p. 96). A continuación Shell cita un pasaje de La religión dentro de los límites de la mera razón (RGV): “[p]or disposiciones de un ser entendemos tanto las partes constitutivas requeridas para él como también las formas de su ligazón para ser un ser tal. Son originales si pertenecen necesariamente a la posibilidad de un ser tal; contingentes si el ser sería en sí posible también sin ellas." (RGV, AA 06: 28). Por último, Wood señala que una disposición es "una tendencia global del organismo a desarrollar el conjunto de capacidades mejor adecuadas para llevar a cabo su modo de vida" (Wood, 2006, p. 249). 


\section{Martín Arias Albisu}

introducido a fin de conferir inteligibilidad a la historia humana en su conjunto. Asimismo, tal concepto no pertenece al entendimiento, sino que es una idea de la razón; ${ }^{3}$ más específicamente, debe entendérselo como una idea regulativa de la razón teórica. Volveremos sobre este punto en el apartado IV.1 de este artículo.

Los problemas principales que debemos examinar a continuación son dos. En primer lugar, cuál es la intención de la naturaleza con respecto a la especie humana. En segundo lugar, cuáles son los mecanismos de los que se sirve la naturaleza para aproximar a la especie humana a la finalidad de esta intención.

\section{I.1. La finalidad de la intención de la naturaleza según laG}

En las primeras dos proposiciones de laG, Kant introduce una concepción teleológica de la naturaleza en general y de la naturaleza humana en particular. Afirma que todas las disposiciones naturales de las criaturas están destinadas a desarrollarse plenamente de acuerdo con su finalidad (IaG, AA 08: 18), y que el desarrollo de la disposición racional humana debe ser la finalidad de los esfuerzos del ser humano. Dado que los conocimientos y la ilustración avanzan progresivamente de generación en generación, la mencionada finalidad no puede alcanzarse en el individuo, sino solamente en la especie (IaG, AA 08: 18-19).

Más adelante, Kant señala que solo en una sociedad dotada de una constitución civil perfecta, esto es, que combine la máxima libertad de los ciudadanos con un poder irresistible que haga cumplir las leyes que hacen posible la coexistencia de la libertad de estos ciudadanos, pueden desarrollarse plenamente las disposiciones humanas. Por este motivo, la conformación de una sociedad estructurada por esta constitución es la tarea más importante para la especie humana (laG, AA 08: 22). La naturaleza nos impone únicamente la tarea de aproximarnos progresivamente a esta constitución. De hecho, esta constitución es una idea de la razón práctica y su

\footnotetext{
${ }^{3}$ Kant entiende por idea de la razón, en general, "un concepto necesario de la razón, al que no le puede ser dado ningún objeto congruente en los sentidos." (A327/B383). Acerca de la conformación de un sistema de acciones humanas a partir de la idea rectora de laG, véase laG (AA 08: 29). Véase también el apartado IV.1 del presente trabajo. Acerca del concepto de idea de la razón práctica, véase infra, n. 4.
} 
Kant y la teleología de la naturaleza: acerca de la intención de la naturaleza en Idea para una historia universal en intención cosmopolita y la garantía de la naturaleza en Hacia la paz perpetua

realización plena es imposible. ${ }^{4}$ Efectivamente, el ser humano tiene inclinaciones animales que lo llevan constantemente a desobedecer las leyes que protegen la libertad de sus semejantes, y por tanto precisa de un amo que lo coaccione a cumplir con las mismas. Sin embargo, este amo es también un ser humano y debería ser justo por sí mismo (es decir, incluso sin tener sobre sí a otro amo), lo cual, para Kant, es imposible. Es en este contexto que Kant escribe su famosa sentencia: "de una madera tan retorcida como de la que está hecho el ser humano no puede tallarse nada enteramente recto" (IaG, AA 08: 23. Traducción modificada). Uno de los factores que nos permiten aproximarnos a una constitución civil perfecta es "una buena voluntad". (laG, AA 08: 23).

Ahora bien, se pregunta Kant, ¿de qué sirve aproximarnos a una constitución civil perfecta en el marco de un Estado si las guerras y los onerosos preparativos para las mismas amenazan constantemente con devastar y consumir las fuerzas de los diferentes Estados? El establecimiento de una constitución civil perfecta, piensa Kant, no tendría sentido si no se formase una confederación de Estados dotada de leyes y de un poder coactivo para hacerlas respetar. En esta confederación los conflictos entre los Estados miembros se solucionarían en el marco legal de dicha confederación (laG, AA 08: 24).

En síntesis, el hilo argumentativo de laG es el siguiente: la naturaleza tiene la intención de que el ser humano desarrolle plenamente sus disposiciones, especialmente la racional; tales disposiciones pueden desarrollarse integralmente solo en el marco de Estados estructurados por una constitución civil perfecta (constitución que es una idea, en sí irrealizable, a la que podemos y debemos aproximarnos

\footnotetext{
${ }^{4}$ Cuando expone el concepto de idea de la razón práctica en KrV, Kant ofrece el ejemplo de una constitución perfecta. Tras mencionar la República de Platón, Kant señala: "[u]na constitución de la máxima libertad humana según leyes que hagan que la libertad de cada cual pueda coexistir con la de los otros [...] es, por lo menos, una idea necesaria" (KrV, A316/B373). "Cuanto más concordantes con esta idea fueran la legislación y el gobierno, tanto menos frecuentes serían, por cierto, las penas; y así, pues, es muy razonable (como lo afirma Platón) que si ellos alcanzaran un orden perfecto, no sería necesaria ninguna de éstas. Aunque esto último nunca llegue a realizarse, es, empero, enteramente acertada la idea que instaura a ese maximum como arquetipo, para llevar, de acuerdo con él, la constitución jurídica de los seres humanos cada vez más cerca de la mayor perfección posible. Pues cuál haya de ser el grado máximo, en el cual deba detenerse la humanidad, y cuán grande haya de ser, por tanto, la grieta que necesariamente quede entre la idea y su realización, eso nadie puede ni debe determinarlo, precisamente porque hay libertad, la que puede traspasar cualquier limite dado" (KrV, A317/B373-374. Traducción modificada).
} 


\section{Martín Arias Albisu}

sucesivamente); y la preservación de estos Estados presupone el establecimiento de una confederación de Estados que garantice la paz entre sus miembros. ${ }^{5}$

\section{I.2. Los mecanismos empleados por la naturaleza para realizar su intención según laG}

Los mecanismos de los que, según laG, se sirve la naturaleza para realizar su intención con respecto al ser humano están contenidos en la famosa doctrina kantiana de la "insociable sociabilidad" de la naturaleza humana. En el presente apartado expondremos esta doctrina. Kant comienza por afirmar que el ser humano tiene una tendencia a socializarse, porque en una sociedad "se siente más como ser humano, esto es, siente el desarrollo de sus disposiciones naturales"; pero posee a la vez una inclinación a aislarse porque quiere disponer todo según sus propios intereses y espera que los demás obren de la misma manera. (laG, AA 08: 20-21. Traducción modificada). Es esta resistencia entre los seres humanos la que vence la inclinación de ellos a la pereza y los mueve a desarrollar sus disposiciones. "[P]or medio de la ambición, el afán de dominio o la codicia", el ser humano busca "una posición entre sus congéneres". De esta manera, se pasa de la barbarie hacia la cultura, se desarrollan "poco a poco todos los talentos" y con el decurso del tiempo, van formándose principios éticos, hasta que, finalmente, una sociedad formada naturalmente pueda transformarse en un "conjunto moral" (laG, AA 08: 21).

Ahora bien, debe notarse que la resistencia entre los individuos y la tendencia de los mismos hacia una "salvaje libertad", abandonadas a sí mismas, impedirían la coexistencia entre los individuos. El mecanismo mismo de la insociable sociabilidad hace necesario entonces que estos individuos renuncien a su salvaje libertad y entren en una condición civil, en la cual, en el caso de que la misma sea perfecta, el "antagonismo generalizado de sus miembros" está vinculado con "la más escrupulosa determinación y protección de los límites de esa libertad para que pueda coexistir con la libertad de los demás" (laG, AA 08: 22).

\footnotetext{
${ }^{5}$ Ameriks señala correctamente que en laG no se ofrece una argumentación suficiente acerca del motivo por el cual el desarrollo de la predisposición racional humana tenga que implicar precisamente el establecimiento de una confederación de Estados (Ameriks, 2009, pp. 58-59).
} 
Kant y la teleología de la naturaleza: acerca de la intención de la naturaleza en Idea para una historia universal en intención cosmopolita y la garantía de la naturaleza en Hacia la paz perpetua

Notemos, por último, que el mecanismo de la insociable sociabilidad no sólo es responsable de la formación de una condición civil entre individuos, sino también de la formación de una condición civil entre Estados. Los Estados particulares poseen una "libertad desenfrenada" en lo que respecta a sus relaciones externas, y deben por tanto esperar de los otros Estados los mismos males que forzaron a los individuos a entrar en una condición civil. La naturaleza emplea el mecanismo de la insociable sociabilidad para procurar la paz y la seguridad de los Estados. En este caso, el antagonismo mutuo adopta la forma de guerras y cuantiosos y constantes preparativos para las mismas. Mediante este antagonismo, la naturaleza, tras repetidas devastaciones y calamidades, arrastra a los Estados a formar la confederación de Estados descrita en el apartado anterior. (laG, AA 08: 24).

\section{La argumentación acerca de la garantía la naturaleza en ZeF}

Kant considera que en el curso mecánico de la naturaleza puede descubrirse una finalidad para la humanidad, a saber, la paz perpetua. Tal finalidad de la naturaleza coincide con el deber moral de producir la condición pacífica en cuestión (ZeF, AA 08: 361-362). Como veremos en el apartado II.2, el reconocimiento de una garantía de la naturaleza para la aproximación a la paz perpetua propicia el deber moral de realizar esta condición, dado que la garantía en cuestión pone en evidencia que un estado de paz perpetua no es una mera quimera.

\section{II.1. El escenario preparado por la naturaleza para la humanidad según ZeF}

Kant comienza su argumentación tratando el tema de la organización que la naturaleza prepara para el ser humano. En esta organización el ser humano es tomado como una especie animal. En primer lugar, la naturaleza se ha ocupado de que los seres humanos puedan poblar todas las partes de la Tierra. Kant presenta ejemplos de esta tesis. Reproducimos únicamente dos de los mismos. 1) Debajo del océano glacial se encuentra el musgo que requiere el reno, animal que, a su vez, sirve de transporte y alimento a los humanos nativos. 2) El camello ocasiona la impresión de haber sido creado para atravesar los desiertos (ZeF, AA 08: 363). 


\section{Martín Arias Albisu}

En segundo lugar, además de posibilitar que los seres humanos vivan en todas las regiones de la Tierra, la naturaleza ha querido que ellos deban vivir en esas regiones, incluso las más apartadas. El medio del que se ha servido la naturaleza para obtener tal fin es la guerra. Efectivamente, los conflictos bélicos, piensa Kant, tienen como resultado un distanciamiento entre los diferentes grupos de seres humanos que han entablado tales conflictos (ZeF, AA 08: 363-365).

En tercer lugar, también por medio de la guerra, la naturaleza ha constreñido a los seres humanos "a entrar en relaciones más o menos legales" (ZeF, AA 08: 363). En el siguiente pasaje Kant expone el punto tercero.

[E]l primer instrumento de guerra de entre todos los animales es el caballo, que el ser humano había aprendido a domar y a domesticar en la época del poblamiento de la tierra [...], así como el arte de cultivar ciertas clases de hierbas [...] Ilamadas cereales [...], sólo podía darse en la condición de Estados ya establecidos, donde tuvo lugar una propiedad de la tierra garantizada, después de que los seres humanos, antes en libertad sin leyes, hubieran sido llevados de la vida de la caza, la pesca y el pastoreo a la vida de la agricultura, y entonces la sal y el hierro fueron descubiertos, tal vez los primeros artículos más ampliamente buscados de un tráfico comercial de distintos pueblos, por lo cual ellos primeramente fueron llevados a una relación pacífica recíproca y así incluso con los más lejanos a un acuerdo, comunidad y relación pacífica mutua (ZeF, AA 08: 363-364. Traducción modificada).

Flikschuh señala acertadamente que el proceso de surgimiento de "comunidades políticas permanentes" descrito en el pasaje citado hace referencia a las "relaciones más o menos legales" aludidas en el tercer punto, y que parecería que la guerra entre "hordas nómadas" contribuyó al surgimiento mencionado (Flikschuh, 2006, p. 389). Además, Flikschuh estima, también correctamente, que los dos primeros ordenamientos de la naturaleza para el ser humano, en la medida en que se refieren a condiciones "basadas materialmente", son compatibles con una consideración de la humanidad como especie animal. "Por el contrario, la entrada en condiciones más o menos legales de derecho positivo indica la emergencia gradual de relaciones 
Kant y la teleología de la naturaleza: acerca de la intención de la naturaleza en Idea para una historia universal en intención cosmopolita y la garantía de la naturaleza en Hacia la paz perpetua

razonadas y, en tanto tal, la transición del estatus animal al racional" (Flikschuh, 2006, p. 390).

\section{II.2. La finalidad y los mecanismos de la naturaleza con respecto a las tres esferas del derecho público según ZeF}

A continuación, Kant presenta un problema diferente. Ya no se trata de identificar la finalidad de la naturaleza para con el ser humano considerado como una especie animal. En cambio, se tiene que considerar la relación de la finalidad de la naturaleza con el fin que la razón práctica impone como deber, a saber, la paz perpetua, y, así, mostrar cómo la naturaleza

suministra la garantía de que aquello que el ser humano debería hacer según las leyes de la libertad, pero que no hace, queda asegurado de que lo hará sin que la coacción de la naturaleza dañe esta libertad, y precisamente según las tres relaciones del derecho público, el derecho político, el derecho de gentes y el derecho cosmopolita (ZeF, AA 08: 365. Traducción modificada).

Expongamos a continuación la relación entre la finalidad de la naturaleza y las tres diferentes esferas del derecho público. Comenzamos con el derecho político (Staatsrecht). Aunque un Estado no se forme con el motivo de terminar las discordias internas, podría formarse, según la garantía de la naturaleza, desde fuera mediante la guerra. En otras palabras, un pueblo se encuentra ante otros y tiene que transformarse en Estado a fin de estar preparado contra ellos como una potencia.

Por otro lado, Kant considera que las repúblicas son los únicos Estados enteramente adecuados al derecho de los seres humanos. Las repúblicas se caracterizan principalmente por la separación entre el poder legislativo y el ejecutivo y por el hecho de que en ellas los ciudadanos legislan indirectamente por medio de sus representantes. Nos interesa destacar que la formación de una república no requiere ciudadanos que fuesen como ángeles, sino que esta puede mantenerse pacíficamente incluso pese a haber sido formada por un pueblo de demonios dotados de entendimiento. Este funcionamiento de las repúblicas es garantizado por la naturaleza, 


\title{
Martín Arias Albisu
}

dado que presupone las tendencias egoístas de los individuos. El problema, afirma Kant, es

\begin{abstract}
ordenar una muchedumbre de seres racionales que, para su conservación, exigen conjuntamente leyes universales, aun cuando cada uno tiende secretamente a eludirlas, y establecer su constitución de modo tal que, aunque en sus disposiciones privadas se esfuercen unos contra otros, estas se contengan mutuamente de manera tal que en su conducta pública el resultado es justamente el mismo que si ellos no tuvieran tales disposiciones malas (ZeF, AA 08: 366. Traducción modificada). ${ }^{6}$
\end{abstract}

Parece claro que, según Kant, para formar una república bastan, por un lado, el temor al castigo consiguiente al quebrantamiento de las leyes y, por el otro, el interés propio de los individuos, interés cuya prosecución y protección presuponen la limitación mutua de las libertades de los individuos, limitación garantizada por una constitución en vigencia. Cabe suponer que las constituciones de las repúblicas, como las de todos los Estados, son inicialmente imperfectas.

Consideremos ahora el derecho de gentes (Völkerrecht). Tal derecho presupone la separación de muchos Estados vecinos. Esta es una situación de guerra. Sin embargo, Kant la considera preferible a la fusión de los diferentes Estados por parte de una monarquía universal conquistadora. La naturaleza se sirve de dos medios para evitar la fusión de los pueblos, a saber, las diferencias de lengua y de religión. Estas diferencias pueden generar conflictos en un primer momento, pero con el avance de la cultura se llega a un amplio acuerdo referido a principios comunes, de modo tal que se obtiene una paz fundada en un equilibrio de fuerzas "en la más viva competencia" (ZeF, AA 08: 367. Traducción modificada). Por otro lado, si bien Kant no toca el tema cuando expone el derecho de gentes, sino cuando trata el derecho político, consideramos que presenta también al antagonismo entre los Estados como un

\footnotetext{
${ }^{6}$ Höffe señala que los demonios mencionados por Kant son “egoístas decididos o radicales” y están guiados por un "interés propio ilustrado". "Donde esperen una ventaja mutua, los demonios cooperarán entre sí; en todos los otros casos serán inescrupulosos al ignorar los intereses de otras personas, tendiendo resueltamente hacia la deshonestidad y el engaño" (Höffe, 1992, 125).
} 
Kant y la teleología de la naturaleza: acerca de la intención de la naturaleza en Idea para una historia universal en intención cosmopolita y la garantía de la naturaleza en Hacia la paz perpetua

mecanismo de la naturaleza que propicia la formación de una federación de Estados (ZeF, AA 08: 366-367. Véase III.d).

Por último, examinemos el derecho cosmopolita (Weltbürgerrecht). La naturaleza une a diversos pueblos, a los cuales el derecho cosmopolita no habría podido proteger contra la guerra, mediante el expediente del provecho recíproco de los mismos. Estamos ante lo que Kant denomina "el espíritu del comercio". Efectivamente, el comercio permite establecer relaciones pacíficas entre diferentes partes del mundo y es incompatible con una situación de guerra. Por tanto, a fin de asegurar su provecho propio, los Estados se ven forzados a evitar la guerra mediante negociaciones.

III. Análisis de las diferencias entre la intención de la naturaleza según laG y la garantía de la naturaleza según ZeF

a. El carácter teórico de la idea de laG y el carácter práctico de la garantía de ZeF. Lo primero que debe notarse es que la intención de la naturaleza de laG, esto es, lo que determina el plan de la historia humana, es una idea de la razón teórica. De hecho, el objetivo de esta idea es dotar de la máxima inteligibilidad sistemática posible a la historia de la humanidad. En cambio, en ZeF, la doctrina kantiana es más compleja. Al final de su exposición de los mecanismos de la naturaleza referidos a las tres esferas del derecho público, Kant afirma:

[d]e esta suerte garantiza la naturaleza la paz perpetua mediante el mecanismo mismo de las inclinaciones humanas; por cierto con una seguridad que no es suficiente para predecir (teóricamente) el futuro de la misma, pero en intención práctica sí es suficiente y convierte en un deber el trabajar con miras a este fin (no meramente quimérico) (ZeF, AA 08: 368. Traducción modificada).

Parecería que la garantía de la naturaleza de ZeF no es formulada con intención teórica, sino práctica (véase Hoesch, 2015). Ciertamente, si bien esta garantía presupone un conocimiento de ciertos mecanismos de la naturaleza, ella no es formulada a fin de obtener un conocimiento teórico de los mecanismos en cuestión, 


\section{Martín Arias Albisu}

sino a fin de propiciar la finalidad moral de aproximarse a la condición de una paz perpetua. Se llega a decir que esta garantía "convierte en un deber el trabajar con miras" al fin de la paz perpetua. Tal vez esta tesis deba entenderse en el sentido de que la garantía de la naturaleza favorece el deber moral impuesto por la razón práctica al mostrar que el fin del mismo es realizable ("no meramente quimérico"). ${ }^{7}$ Efectivamente, antes de comenzar la exposición mencionada más arriba, esto es, la de los mecanismos naturales en su relación con los tres dominios del derecho público, Kant afirma:

[a]hora bien, la cuestión que concierne a lo esencial del propósito de la paz perpetua es: lo que la naturaleza hace en esta intención en relación con el fin que su propia razón convierte en un deber para el ser humano, por lo tanto para favorecer [zu Begünstigung] su intención moral (ZeF, AA 08: 365. Traducción modificada). ${ }^{8}$

Por último, notemos que el hecho de que la paz perpetua no sea una quimera no implica que esta paz sea integralmente realizable, dado que es una idea de la razón, sino que es posible acercarse a ella asintóticamente. De hecho, Kant asevera:

[s]i existe un deber y al mismo tiempo una esperanza fundada de que hagamos realidad el estado de un derecho público, aunque sólo en una aproximación que

\footnotetext{
${ }^{7}$ El hecho de que la tesis en cuestión no puede comprenderse en el sentido de que la garantía de la naturaleza misma ocasiona un deber moral se pone en evidencia en el siguiente pasaje: "cuando digo de la naturaleza que ella quiere que suceda esto o aquello, esto no quiere decir que ella nos imponga un deber de hacerlo (pues esto sólo puede hacerlo la razón práctica libre de coacción), sino que ella misma lo hace, querámoslo nosotros o no". (ZeF, AA 08: 365. Traducción modificada).

${ }^{8}$ Cabe destacar que, si bien la idea rectora de laG pertenece a la razón teórica, existen al menos dos utilidades prácticas que resultan de la comprensión de la historia hecha posible por la idea en cuestión: 1) la difusión de la idea de laG entre el público ilustrado puede favorecer la aproximación a la finalidad de la historia contenida en dicha idea (IaG, AA 08: 28); 2) la perspectiva de la realización de esta finalidad proporciona esperanza al agente moral (IaG, AA 08: 30). Remarquemos que esto no implica que la idea de laG sea de naturaleza práctica. En pocas palabras, esta idea es formulada para comprender globalmente la historia, y esta compresión global tiene dos utilidades prácticas. Como afirma Wood (2009), "[e]n este ensayo [Autor: laG], el tema es la historia humana, y la idea en cuestión es por lo tanto un idea de máxima inteligibilidad teórica para la historia humana. Los resultados de este proyecto pueden converger [...] con nuestros esfuerzos prácticos, motivados por consideraciones morales. Pero considerar el proyecto principal de Kant en Idea para una historia universal como motivado por la moralidad es malinterpretar totalmente el ensayo desde el comienzo" (p. 112. Cf. Wood, 2006, pp. 243-247, 255-258).
} 
Kant y la teleología de la naturaleza: acerca de la intención de la naturaleza en Idea para una historia universal en intención cosmopolita y la garantía de la naturaleza en Hacia la paz perpetua

progrese hasta el infinito, entonces la paz perpetua [...] no es una idea vacía sino una tarea que, resuelta poco a poco, se acerca continuamente a su meta. (ZeF, AA 08: 386. Traducción modificada).

b. El lugar de la condición de paz en las argumentaciónes de laG y ZeF. En segundo lugar, existe una diferencia entre el lugar que el ideal de la paz perpetua ocupa en las dos diferentes argumentaciones de laG y ZeF. En laG, la paz entre los Estados es una condición de la preservación de una constitución civil perfecta (en sí irrealizable, si bien debemos aproximarnos progresivamente a ella), constitución que, a su vez, es una condición del desarrollo pleno de las disposiciones humanas, desarrollo que es la intención de la naturaleza. En cambio, en ZeF, la paz perpetua es presentada como una finalidad impuesta por la razón práctica. Esta paz es una idea de la razón que, en virtud de su naturaleza, es en sí integralmente irrealizable. Tenemos el deber de aproximarnos progresivamente a esta idea y existe una garantía de la naturaleza para la mencionada aproximación.

c. Las diferentes concepciones del Estado de laG y ZeF. Como vimos en el apartado I.1, en laG Kant afirma que los seres humanos tienen una inclinación hacia una libertad salvaje. Por tanto, requieren de un amo que los constriña a obedecer las leyes que permiten y garantizan la coexistencia de los individuos. Ahora bien, este amo de la sociedad no puede sufrir coacción para obrar de una manera conforme a derecho, por lo cual debería ser al mismo tiempo humano y justo, lo cual para Kant es imposible. Por estos motivos, Kant considera que el problema de la formación de una sociedad estructurada en función de una constitución civil perfecta no posee una solución integral. Una de las condiciones para la aproximación a dicha constitución es una buena voluntad. (IaG, AA 08: 23).

En ZeF, como se vio en el apartado II.2, Kant afirma en cambio que la constitución republicana es la que más se ajusta al derecho humano y que su realización es factible. Se dice allí que la conformación de una república no sólo no requiere ángeles, sino que también es viable a partir de un pueblo de demonios dotados de entendimiento. A diferencia de laG, entonces, en ZeF no se afirma que se 


\section{Martín Arias Albisu}

requiera buena voluntad para conformar un Estado justo y pacífico. La perspectiva de Kant parece ser que ciertas características de los Estados republicanos, consistentes en la protección del interés de cada ciudadano y en los castigos a las transgresiones a las leyes, bastan para que un individuo poseído de malas inclinaciones se comporte como un buen ciudadano.

\section{d. Las diferentes concepciones de las asociaciones de Estados de laG y ZeF.} Existen al menos dos diferencias entre las concepciones de asociaciones de Estados de laG y ZeF. La primera de ellas se refiere a la índole de las asociaciones, mientras que la segunda está vinculada con la argumentación desarrollada por Kant para proponerlas.

En el primer texto, la necesidad de formar una confederación de Estados se deriva de la concepción de la insociable sociabilidad implantada en el ser humano por la intención de la naturaleza. El objetivo de la intención de la naturaleza al conformar este mecanismo es el desarrollo pleno de las disposiciones humanas, especialmente la racional. La confederación de Estados propuesta posee leyes y un poder coactivo capaz de hacerlas cumplir.

En ZeF, Kant considera la figura de un Estado mundial similar al de laG. Sin embargo, estima que este Estado es irrealizable y propone por tanto, como sucedáneo, una liga de Estados sin leyes públicas ni poder coactivo (ZeF, AA 08: 354-357). ${ }^{9}$ Por

\footnotetext{
${ }^{9}$ Según Kant, los individuos en estado de naturaleza tienen que salir del mismo para entrar en una condición civil, dado que toda condición civil es mejor que la ausencia de la misma. En cambio, los Estados están en estado de naturaleza sólo en lo que hace a sus relaciones externas y albergan dentro de sí una condición civil de cierto grado de perfección. Por tanto, los Estados individuales no pueden ser forzados a formar parte de un Estado mundial, porque ello podría implicar la destrucción de los derechos asegurados por la condición civil de estos Estados individuales (ZeF, AA 08: 355-356. Seguimos la explicación de este argumento presentado por Kleingeld, 2004, pp. 308-310. Cf. Williams, 1983, p. 265). Kleingeld ofrece también una interesante explicación del cambio de postura de Kant en ZeF, la cual está en consonancia con la exposición que acabamos de presentar. En ZeF aparecen dos cuestiones que estaban ausentes en laG. En primer lugar, la tendencia pacífica de las repúblicas. Efectivamente, en el marco de una república son los ciudadanos mismos, mediante sus representantes, quienes deben consentir en iniciar las acciones bélicas. Es evidente que en este caso los ciudadanos serían reticentes a emprender tal empresa, dado que tendrían que hacerse cargo de todas las penurias de la guerra, tales como pagar los gastos de la misma, encargarse de la devastación que ella provoca y emprender ellos mismos la lucha. En cambio, en un Estado que no es republicano, el jefe del Estado no es un integrante del mismo, sino su propietario. Por tanto, es fácil para este jefe decidir iniciar acciones bélicas, dado que esta decisión no lo obliga a renunciar a ninguno de sus privilegios (ZeF, AA 08: 350). En segundo lugar, la factibilidad de conformar las repúblicas, incluso si los ciudadanos están desprovistos de una buena voluntad (ZeF, AA 08: 350. Véase III.c). Kleingeld concluye que esta evolución del pensamiento kantiano
} 
Kant y la teleología de la naturaleza: acerca de la intención de la naturaleza en Idea para una historia universal en intención cosmopolita y la garantía de la naturaleza en Hacia la paz perpetua

otro lado, llama la atención el que, en el "Segundo artículo definitivo para la paz perpetua" de ZeF (ZeF, AA 08: 354-357), donde Kant examina la temática de la asociación de Estados, no se mencione la garantía de la naturaleza. La argumentación de Kant no comienza con esta garantía, sino con el hecho de que los Estados, en ausencia de toda condición civil en lo que hace a sus relaciones externas, se perjudican ya por su mera coexistencia (ZeF, AA 08: 354). Llama también la atención el que, cuando Kant discute el tema de la garantía de la naturaleza ("Suplemento primero. De la garantía de la paz perpetua"; ZeF, AA 08: 361-368), no trate sustancialmente el problema de la confederación de Estados. Recordemos que, al estudiar el tema de la garantía de la naturaleza referida al derecho de gentes (véase II.2), Kant hace énfasis en los mecanismos de la naturaleza para separar a los diferentes Estados y evitar así la fusión de ellos en una monarquía universal conquistadora. Ahora bien, en un párrafo anterior, también perteneciente al "Suplemento primero. De la garantía de la paz perpetua", en el que está bajo examen la garantía de la naturaleza referida al derecho político, se afirma que "el mecanismo de la naturaleza mediante inclinaciones egoístas, que naturalmente se contrarrestan entre sí también externamente" puede ser empleado como medio por la razón para su fin jurídico al "fomentar y garantizar las paz tanto interna como exterior" (ZeF, AA 08: 366-367. Traducción modificada). Consideramos, en primer lugar, que este texto puede entenderse en el sentido de que uno de los mecanismos de la naturaleza que permite fomentar y garantizar la paz exterior de los Estados es el antagonismo entre los mismos. En segundo lugar, debe notarse que lo que la razón puede emplear es el mecanismo natural vinculado con el contrarrestarse de inclinaciones egoístas, lo cual no implica que esta facultad fomente la intensificación de las inclinaciones egoístas que sean contrarias a la moral (véase IV.3)

explica el hecho de que el filósofo de Königsberg proponga una confederación voluntaria de Estados. Si es cierto que las repúblicas son los únicos Estados justos, entonces Kant tiene que negar la posibilidad de que una república pueda ser obligada a formar parte de un Estado mundial posible y probablemente imperfecto (Kleingeld, 2009, pp. 181-182). Acerca de los argumentos de Kant sobre los problemas involucrados en el concepto de un Estado mundial, véase En torno al tópico: "eso vale para la teoría pero no sirve de nada en la práctica" (TP - 1793-, AA 08: 310-311), ZeF (AA 08: 354-356) y Metafísica de las costumbres (MS -1797-, AA 06: 350). 


\section{Martín Arias Albisu}

e. La función del comercio en laG y ZeF. En laG, Kant señala que, en virtud de los constantes y onerosos preparativos para la guerra por parte de los diferentes Estados, estos se ven obligados a contraer deuda pública. Dado que esta deuda está siempre en aumento, en un futuro la guerra será considerada como una empresa de riesgosa ejecución. Efectivamente, dado el entrelazamiento comercial "en esta parte del mundo", los problemas financieros de un Estado afectan a todos los demás (IaG, AA 08: 28). La interdependencia comercial entre los Estados ocasiona incluso que algunos de estos se presten para arbitrar en los conflictos interestatales. Esta situación prepara la integración de los Estados en un gran cuerpo político, el cual, a su vez, con el tiempo puede transformarse en el Estado mundial propuesto en laG. Por tanto, según laG, la actividad comercial hará necesario que vayan cesando las guerras y que la humanidad se acerque progresivamente a una "condición cosmopolita universal" (IaG, AA 08: 28. Traducción modificada). En ZeF, Kant presenta esta temática con el título de "espíritu del comercio". Este espíritu es parte de la garantía de la naturaleza con respecto al derecho cosmopolita y fue expuesto en II.2. Como vimos, al igual que en laG, la actividad comercial está vinculada con el establecimiento de relaciones pacíficas entre los Estados, dado que esta actividad no puede desarrollarse en una situación de guerra. Si bien en el contexto de esta presentación Kant no hace referencia a una confederación de Estados, cabe suponer que considera que la actividad comercial es una condición propiciadora de la formación de la misma. ${ }^{10}$

\section{Los fundamentos de las concepciones teleológicas de la naturaleza de laG y ZeF}

\section{IV.1. El fundamento de la concepción teleológica de la naturaleza en laG}

Hemos adelantado que, en laG, la idea de la historia es una idea de la razón teórica. Esta idea cumple una función similar a la desempeñada por las ideas regulativas de la razón teórica y, por tanto, debe concebírsela como una idea de esa clase. El concepto de idea regulativa de la razón teórica es presentado en el "Apéndice

\footnotetext{
${ }^{10}$ Caranti sostiene acertadamente que la tesis kantiana acerca de la relación entre comercio y paz tiene un componente objetivo y otro subjetivo. "Respectivamente: (a) el comercio internacional es imposible en tiempos de guerra, mientras que la interdependencia económica entre los estados disminuye la probabilidad de guerra; (b) el hábito de regatear y negociar acostumbra a la gente a resolver sus desacuerdos de una manera no violenta". (Caranti, 2014, p. 147)
} 
Kant y la teleología de la naturaleza: acerca de la intención de la naturaleza en Idea para una historia universal en intención cosmopolita y la garantía de la naturaleza en Hacia la paz perpetua

a la dialéctica trascendental" de KrV (A642/B670ss.). Existen al menos tres tipos de ideas regulativas de la razón teórica. En primer lugar, ideas específicas de las diferentes disciplinas. Estas ideas establecen la "forma de un todo del conocimiento, [un todo] que precede al conocimiento determinado de las partes, y que contiene las condiciones para asignarle a priori a cada parte su lugar y su relación con las restantes". En virtud de esta unidad, el conocimiento, de ser un mero agregado, pasa a ser un sistema interconectado (A645/B673). Esta sistematicidad es una condición necesaria de la cientificidad (A832/B860). La idea de la historia de laG debe concebirse como una idea de esta clase, dado que determina un sistema en el cual se incluyen ordenadamente los distintos materiales del conocimiento histórico.

Aparte de las ideas mencionadas, Kant discute otras dos clases de ideas regulativas de la razón teórica. Estas dos clases de ideas no tienen como campo de aplicación disciplinas específicas, dado que su función es dirigir a todos los conocimientos del entendimiento hacia la mayor unidad y extensión sistemática posible. Para nuestros fines, basta con que nos limitemos a exponer la función regulativa de la idea de Dios. ${ }^{11}$ Cuando explica esta función regulativa, Kant afirma:

\footnotetext{
${ }^{11}$ En la primera parte del "Apéndice a la dialéctica trascendental” de KrV (A642/B670ss.), Kant presenta los principios regulativos de homogeneidad, especificación y continuidad. El primero de ellos ordena buscar la identidad de la especie en distintos objetos empíricos y la identidad del género en distintas especies. De este modo se persigue la máxima unidad de los conocimientos. El principio de especificación manda intentar dividir o especificar los géneros en diferentes especies. De esta manera, se intenta conseguir la máxima extensión de los conocimientos. Por último, el principio de continuidad establece la necesidad de intentar introducir, entre dos especies de un género consideradas preliminarmente como colindantes, cada vez más especies intermedias. En la segunda parte del mencionado "Apéndice" (A669/B697ss.), se expone la función regulativa de las ideas del alma, el mundo y Dios. Según Kant, podemos y debemos representar sistemáticamente los fenómenos al relacionarlos con las ideas en cuestión. Por ejemplo, la idea regulativa del alma permite representar con la mayor sistematicidad posible los fenómenos internos, al considerarlos como determinaciones de una única substancia permanente (a saber, el alma), determinaciones que son así entendidas como diferentes de los fenómenos externos. Debe señalarse que de esta manera no obtenemos un conocimiento de lo que sea el alma en sí misma. Efectivamente, la función regulativa que nos ocupa no presupone una referencia de las ideas del alma, el mundo y Dios a entidades independientes de las mismas. Más bien, los fenómenos son representados sistemáticamente al ser puestos en relación con lo que Kant denomina "objeto en la idea" (A670/B698), esto es, el contenido lógico de las ideas en cuestión considerado como un objeto. Allison propone entender el concepto de objeto en la idea como un objeto intencional (Allison, 2004, pp. 409 y 438). Previamente, Zocher propuso entender este concepto como un “cuasi-objeto" [Quasi-Gegenstand] (Zocher, 1958, 48).
} 
[e]sta unidad formal suprema que se basa solamente en conceptos de la razón, es la unidad de las cosas conforme a fines; y el interés especulativo de la razón torna necesario considerar todo ordenamiento [Anordnung] en el mundo como si hubiese brotado del designio de una razón suprema. Pues tal principio abre nuevas perspectivas a nuestra razón, dirigida al campo de las experiencias, [perspectivas] de conectar las cosas del mundo de conformidad con leyes teleológicas, y de llegar, por ese medio, a la máxima unidad sistemática de ellas (A686-687/B714-715).

La función regulativa de la idea de Dios permite alcanzar la máxima unidad sistemática posible de los fenómenos mediante la introducción de leyes teleológicas. La mencionada unidad conforme a fines se obtiene considerando "todo ordenamiento [Anordnung] en el mundo como si hubiese brotado del designio de una razón suprema". Por tanto, puede afirmarse que la concepción teleológica de la naturaleza de laG no es una presuposición infundada, sino que está fundamentada en el uso regulativo de la razón teórica expuesto en KrV.

En laG, Kant introduce su concepción teleológica de la siguiente manera:

Todas las disposiciones naturales de una criatura están destinadas a desarrollarse alguna vez completamente y con arreglo a un fin. Esto se confirma en todos los animales tanto por la observación externa como por la interna o analítica. Un órgano que no debe ser utilizado, un ordenamiento [Anordnung] que no alcanza su finalidad, supone una contradicción dentro de la doctrina teleológica de la Naturaleza. Y si renunciáramos a ese principio, ya no tendríamos una Naturaleza que actúa conforme a leyes, sino una Naturaleza que no conduce a nada, viniendo entonces a ocupar una desazonante casualidad el puesto del hilo conductor de la razón (IaG, AA 08: 23).

En el pasaje citado se afirma que todas las disposiciones de las criaturas están destinadas a desarrollarse plenamente y de acuerdo con una finalidad. Recordemos que las disposiciones de un ser son, a grandes rasgos, tendencias hereditarias dentro de su especie. La actualización de estas tendencias presupone condiciones ambientales favorables $y$, en el caso del ser humano, queda a cargo del mismo el crear las 
Kant y la teleología de la naturaleza: acerca de la intención de la naturaleza en Idea para una historia universal en intención cosmopolita y la garantía de la naturaleza en Hacia la paz perpetua

condiciones para el desarrollo óptimo de las mismas (véase supra, n. 2). Toda esta concepción es parte de "la doctrina teleológica de la naturaleza".

Llama la atención el que en los dos últimos pasajes citados Kant se sirva del término "Anordnung", esto es, ordenamiento, como ejemplo de un elemento poseedor de finalidad. Además, en otro pasaje del "Apéndice" de KrV, Kant, al igual que en el pasaje antes citado de laG, hace referencia a la finalidad de los órganos animales ("un miembro de un cuerpo animal" A686/B714). Estos usos terminológicos sustentan nuestra tesis según la cual la concepción teleológica de la naturaleza de laG es fundamentada por la doctrina del uso regulativo de las ideas de la razón teórica de KrV. Ahora bien, el estudio del problema de si la doctrina en cuestión es justificada suficientemente por la argumentación kantiana excede los límites del presente trabajo. $^{12}$

\section{IV.2. El fundamento de la concepción teleológica de la garantía de la naturaleza en}

\section{ZeF}

Al comienzo del "Suplemento primero. De la garantía de la paz perpetua" de ZeF, Kant introduce el concepto de una garantía de la naturaleza. Citamos el pasaje a continuación.

Aquello que suministra esta garantía es, nada menos, que la gran artista de la naturaleza (natura daedala rerum), en cuyo curso mecánico brilla visiblemente una finalidad: que mediante la discordia de los seres humanos pueda surgir la armonía,

\footnotetext{
${ }^{12}$ La tesis de que la teleología de la naturaleza de laG es fundamentada por la concepción de las ideas regulativas de la razón teórica de $\mathrm{KrV}$ es defendida por Kleingeld (2008 y 2009). En cambio, Allison (2009) considera que esta teleología no queda realmente fundamentada sino hasta la publicación de la Crítica de la facultad de juzgar en 1790. Fiegle (2014: 169) sostiene implícitamente una tesis similar a la de Allison. También, aunque explícitamente, lo hace Vaki (2014: 196). Sin embargo, más adelante en este mismo trabajo, Vaki también sostiene que la "teleología de la naturaleza" debe ser entendida como una idea regulativa de la razón (2014: 204). Con respecto a este último punto, la posición de Vaki es compleja. Sostiene que, desde la perspectiva del historiador, la idea de laG es una idea regulativa de la razón como las de $\mathrm{KrV}$, mientras que desde el punto de vista del agente, es un postulado de la razón práctica que motiva los esfuerzos por realizar el bien supremo dentro de la historia (2014: 206). Un postulado de la razón práctica es "una proposición teórica, pero que como tal no puede ser demostrada, en cuanto depende inseparablemente de una ley práctica que tiene una validez incondicionada a priori" (Crítica de la razón práctica $=\mathrm{KpV}, \mathrm{AA}$ 05: 122. Traducción modificada). Ejemplos de ellos son la libertad de la voluntad, la inmortalidad del alma, y la existencia de Dios. Debe notarse, contra Vaki, que este concepto de postulado no aparece en laG.
} 


\section{Martín Arias Albisu}

incluso contra su voluntad; y por tal razón es llamada destino, como necesitación de una de sus leyes de eficiencia según una causa desconocida para nosotros; pero en consideración a su finalidad en el curso del mundo, como la sabiduría profunda de una causa más elevada dirigida hacia el fin final objetivo del género humano y que predetermina este curso del mundo, es llamada providencia; que nosotros por cierto no podemos propiamente reconocer en estos artificios de la naturaleza, o siquiera inferirla a partir de ellos, sino que (como en toda relación de la forma de las cosas con sus fines en general) sólo podemos y tenemos que añadirla en el pensamiento, para hacernos un concepto de su posibilidad según la analogía con las acciones del arte humano. (ZeF, AA 08: 360-362. Traducción modificada).

Kant afirma que la causa de la "finalidad en el curso del mundo" no puede ser reconocida en "los artificios de la naturaleza" ni inferida a partir de ellos. Más bien, sólo podemos y tenemos que añadirla con el pensamiento "para hacernos un concepto de su posibilidad según la analogía con las acciones del arte humano", "(como en toda relación de la forma de las cosas con sus fines en general)". Esta afirmación hace referencia a los desarrollos de la "Crítica de la facultad de juzgar teleológica" de la Crítica de la facultad de juzgar (KU, 1790). Según este texto, a fin de enjuiciar a los productos organizados de la naturaleza como fines internos de la misma, debemos emplear una analogía con la causalidad conforme a fines del arte humano. Lo distintivo de esta causalidad es que el concepto de un efecto sirve de finalidad de la acción causal que produce al efecto tal. Por ejemplo, el concepto de una manzana por la que tengo apetito me lleva a producir el estado de cosas consistente en mi apoderamiento de la manzana.

El mencionado enjuiciamiento de los seres organizados de la naturaleza no proporciona un conocimiento explicativo, sino que

se aplica con derecho a la investigación de la naturaleza, al menos problemáticamente; pero sólo para ponerla en analogía con la causalidad conforme a fines bajo principios de observación [Beobachtung] e indagación [Nachforschung], sin pretender explicarla [erklären] de acuerdo con ello (KU, AA 05: 360. Traducción modificada). 

de la naturaleza en Hacia la paz perpetua

El mencionado modo de juzgar corresponde a lo que Kant denomina "facultad de juzgar reflexionante" ${ }^{13}$

En KU, a la facultad de juzgar reflexionante se le atribuyen principalmente tres funciones: 1) la representación de la naturaleza como un sistema de leyes empíricas particulares conformado como si hubiese sido diseñado con la finalidad de concordar con nuestra facultad cognoscitiva; 2) la formulación de juicios estéticos; y 3) la formulación de los juicios acerca de la teleología de la naturaleza. Aquí nos interesan fundamentalmente estos últimos juicios. Señalemos que en ninguno de estos tres casos puede la facultad de juzgar reflexionante producir conocimiento objetivo. Más bien, se trata de modos de enjuiciamiento referidos exclusivamente a la peculiar constitución de nuestra facultad cognoscitiva. ${ }^{14}$

Como todos los seres organizados, el ser humano es un fin de la naturaleza. Ahora bien, la facultad de juzgar reflexionante no sólo considera a los seres organizados como fines, sino que también concibe a la naturaleza entera como un sistema de fines cuyo fin último [letzter Zweck] es el ser humano (KU, AA 05: 429). El ser humano es este fin último porque es el único ser en la Tierra que puede proponerse fines (KU, AA 05: 430). La cultura, en tanto “[p]roducción de la aptitud de

\footnotetext{
${ }^{13}$ Kant considera que, en un objeto que es un fin interno de la naturaleza, las partes deben ser posibles únicamente en su relación con el todo. Efectivamente, el objeto mismo es un fin, y se encuentra "bajo un concepto o una idea que ha de determinar a priori todo cuanto está contenido en ella." Los objetos que sólo pueden pensarse de esta manera son obras del arte, y son producidos por una causa racional distinta de y exterior al producto de la naturaleza, causa racional que contiene la idea del todo del producto en cuestión. Ahora bien, si es que ha de haber productos naturales que tengan en sí mismos relaciones con fines, en lo que hace a la posibilidad interna de los primeros y no meramente mediante una relación con la causalidad externa de "los conceptos de seres racionales", se requiere que las partes del producto en cuestión "queden enlazadas en la unidad de un todo por el hecho de que cada una de tales partes sea recíprocamente causa y efecto de su forma". Efectivamente, piensa Kant, sólo de esta manera es posible que "la idea del todo determine a su vez la forma y el enlace de todas las partes: no como causa -porque entonces sería un producto del arte-, sino como fundamento cognoscitivo, para quien lo enjuicie, de la unidad sistemática de la forma y del enlace de toda la diversidad contenida en la materia dada." (KU, AA 05: 373).

${ }^{14}$ Debe notarse que los juicios estéticos son más "subjetivos" que la representación sistemática de la naturaleza y los juicios teleológicos. En los dos últimos casos, aunque el enjuiciamiento es conforme a nuestras facultades cognitivas y no hay un conocimiento objetivo, hay efectivamente una referencia a un elemento objetivo (las leyes particulares de la naturaleza y los fines internos de esta última). En cambio, en los juicios estéticos hay solamente una referencia a un sentimiento propio del sujeto, sentimiento carente de toda referencia a objetos.
} 


\section{Martín Arias Albisu}

un ser racional para cualesquiera fines en general", es aquello en el ser humano que lo convierte en el mencionado fin último (KU, AA 05: 431).

Asimismo, el ser humano no es solamente el fin último de la naturaleza, sino también el fin final [Endzweck] de la creación, en la medida en que es un ser moral. Considerado como noúmeno, esto es, como libre y dotado de una causalidad por libertad, el ser humano es incondicionado, es decir que, a diferencia de los otros entes naturales, no depende de una condición diferente. Todos los entes naturales están subordinados al ser humano en tanto ser moral. (KU, AA 05: 434-435).

En el $\S 63$ de KU se presenta el concepto de "finalidad relativa", mientras que en el §82 de la misma obra se expone una finalidad similar denominada "externa". Estas finalidades consisten, a grandes rasgos, en la utilidad que, en tanto medio, un ser natural reporta a otro ser natural en tanto fin. En el §82, Kant indica que los seres naturales que no poseen finalidad interna, como el aire y el agua, pueden servir de medios para otros seres, siempre y cuando estos últimos sean seres organizados, a saber, fines de la naturaleza. (AA 05: 425). Con respecto a los fines de la naturaleza, debe notarse que, si la finalidad de la existencia de un ser natural se encuentra dentro del mismo, entonces es un fin final; en cambio, si la finalidad de la existencia de un ser natural se encuentra en un ser natural fuera del mismo, entonces sirve también como medio y no es un fin final. Ahora bien, dentro del ámbito de la naturaleza no se encuentra un fin final (KU, AA 05: 426). Como hemos visto, este fin final de la creación es el ser humano considerado como noúmeno.

Debe notarse que algunos ejemplos de finalidad relativa ofrecidos en el $\S 63$ de $\mathrm{KU}$ son retomados en la exposición de ZeF. En el $\$ 63$ de $\mathrm{KU}$ se dice que los renos "encuentran suficiente alimento en un musgo seco que ellos mismos tienen que escarbar bajo la nieve" y se dejan domar fácilmente (AA 05: 369. Traducción modificada). Como vimos en el apartado II.1, Kant menciona este ejemplo en ZeF ("es admirable que en los fríos desiertos junto al océano glacial crezca todavía el musgo que, bajo la nieve, escarba el reno para ser él mismo el alimento o también el medio de transporte de los ostiakos y samoyedos"; AA 08: 363. Traducción modificada). 
Kant y la teleología de la naturaleza: acerca de la intención de la naturaleza en Idea para una historia universal en intención cosmopolita y la garantía de la naturaleza en Hacia la paz perpetua

Puede afirmarse que la argumentación en torno a la garantía de la naturaleza se sirve, en un primer momento, de la noción de finalidad relativa o externa introducida en KU que acabamos de exponer. Efectivamente, Kant considera, como vimos, que ciertos seres de la naturaleza reportan utilidad al ser humano considerado, por un lado, como especie animal y, por el otro, como un ser dotado de razón práctica. En el primer caso, los ordenamientos de los que se ha servido la naturaleza para conseguir la finalidad de que el ser humano habite en todo el mundo y de manera comunitaria son: 1) la presencia en toda la Tierra de condiciones suficientes para el sustento del ser humano; 2) las guerras y el consiguiente desplazamiento de los bandos involucrados en el conflicto hacia todas las regiones de la Tierra; y 3) el establecimiento, mediante la guerra, de relaciones "más o menos legales" entre los individuos (véase II.1). En el segundo caso, como también vimos, la naturaleza emplea diferentes mecanismos para aproximar progresivamente al ser humano a la idea de la paz perpetua, y Kant examina estos mecanismos en su relación con las tres esferas del derecho público (el derecho político, el derecho de gentes y el derecho cosmopolita). Tales mecanismos son, respectivamente: 1.1 ) la guerra como causa de que los pueblos se conviertan en Estados para poder ofrecer resistencia a los demás; 1.2) la persecución del interés propio y el miedo al castigo subsiguiente al quebrantamiento de una ley, a fin de formar un Estado pacífico; 2.1) el antagonismo mutuo como causa propiciadora de una asociación de Estados (véase supra, III.d); 2.2) las diferencias de lengua y religión para que los Estados individuales no sean fusionados en y por una monarquía despótica y conquistadora; y 3) la búsqueda del provecho propio recíproco implícita en la institución del comercio para establecer relaciones pacíficas entre diferentes partes del mundo (véase II.2).

En un segundo momento, parecería que Kant, en lo que respecta a los mecanismos de la naturaleza para aproximar al ser humano a la condición de una paz perpetua, se sirve implícitamente de su concepción del ser humano como fin final de la creación. En efecto, los mecanismos naturales mencionados son factores que propician la paz dentro de un Estado y en las relaciones interestatales. Y producir tal condición de paz es un deber impuesto por la razón práctica, esto es, que se origina en último 


\section{Martín Arias Albisu}

término en el ser humano como homo noumenon. En pocas palabras, los mecanismos en cuestión favorecen el cultivo de la moralidad humana y el requerimiento de paz de la misma. Por tanto, pueden concebirse como medios para el fin final de la creación.

\section{IV.3. Teleología y libertad}

La argumentación de los últimos dos apartados lleva a plantear dos interrogantes. En primer lugar, ¿puede tan sólo la naturaleza emplear medios inmorales para obtener la paz, tales como la guerra, o también los seres humanos pueden servirse de ellos con el mismo fin? En segundo lugar, ¿en qué medida los mecanismos que pertenecen a la naturaleza pueden vincularse con el libre albedrío humano ${ }^{15}$

Con respecto al primer punto, debe notarse que los mecanismos de la naturaleza no son considerados por la razón práctica como medios necesarios para obtener la paz. La razón práctica ordena inmediatamente la abolición total de la guerra $y$, por tanto, prescribe la realización de una de las condiciones de la paz perpetua según ZeF, a saber, que los Estados sean republicas, dado que ellas son más proclives a la paz (AA 08: 349-353. Véase supra, n. 10). Es únicamente la naturaleza, teleológicamente concebida, la que implanta en los individuos tendencias egoístas a fin de que, entre otras cosas, lleven a la formación de una república. Con respecto a este punto, nos parece acertada una observación de Wood. Si bien Wood formula esta tesis en el marco de un estudio del concepto de insociable sociabilidad presentado en laG (véase I.2), nos parece también aplicable a la problemática de ZeF. Estimo que la tesis principal de Wood es que hay un punto de quiebre en la historia humana. Recordemos que, según Kant, el antagonismo entre los individuos permite pasar de la barbarie a la cultura, desarrollar los talentos y posibilitar la formulación de principios éticos (laG, AA 08: 21). De manera similar, en ZeF (AA 08: 360), como vimos en el

\footnotetext{
15 “[U]n albedrío es sensible en la medida en que es afectado patológicamente (por móviles de la sensibilidad); se denomina animal (arbitrium brutum) cuando puede ser coaccionado patológicamente. El albedrío humano es, ciertamente, un arbitirum sensitivum, pero no es brutum, sino liberum, porque la sensibilidad no hace necesaria la acción de él, sino que en el ser humano reside una facultad de determinarse por sí mismo, independientemente de la coacción ejercida por móviles sensibles" (A534/B562).
} 
Kant y la teleología de la naturaleza: acerca de la intención de la naturaleza en Idea para una historia universal en intención cosmopolita y la garantía de la naturaleza en Hacia la paz perpetua

apartado IV.2, Kant afirma que la naturaleza quiere "que mediante la discordia de los seres humanos pueda surgir la armonía". En consonancia con estas afirmaciones, Wood sostiene que el antagonismo mencionado es una condición del reconocimiento pleno de la ley moral por parte de los individuos. Ahora bien, el contenido de la ley moral no puede ser determinado por este antagonismo, dado que este contenido es $a$ priori. Además, ese contenido está en directa contradicción con el mismo antagonismo que es causa de su reconocimiento pleno. Efectivamente, la ley moral nos ordena renunciar a ese antagonismo. Wood (2009) concluye:

esto significa que tenemos que esforzarnos por el bien propiamente humano no meramente de manera independiente de las mismas condiciones en nuestra propia naturaleza que hicieron posible para nosotros la conciencia racional de este bien, sino incluso en conflicto directo con ellas (p. 123. Cf. Wood, 2006, pp. 252ss.).

Según entendemos, el punto de quiebre en la historia que puede leerse en la interpretación de Wood coincide con el paso del antagonismo como medio para el reconocimiento íntegro de la ley moral a la limitación de este antagonismo impuesta por la ley moral. Aplicando esta interpretación al surgimiento de los Estados, podemos afirmar que, en un primer momento, la insociable sociabilidad humana (laG) o la búsqueda exclusiva del interés propio (ZeF) lleva a establecer una condición civil, en sus inicios rudimentaria, cuyo objetivo es garantizar y asegurar la protección de la esfera de libertad de cada ciudadano. Sin esta condición civil, los individuos no podrían disfrutar "los frutos de sus esfuerzos", lo cual conduciría a socavar "los incentivos mismos para el desarrollo de nuestras predisposiciones que la insociable sociabilidad se supone que debe promover" (Wood, 2009, 116). Con el desarrollo de la cultura, la civilización y especialmente la moralidad, este orden civil es apreciado y mejorado gracias a la buena voluntad de sus ciudadanos.

La conclusión que podemos extraer es que los mecanismos de la naturaleza para obtener la paz no son necesariamente morales, y que llega un punto en el desarrollo de estos mecanismos en el que los seres humanos reconocen plenamente su razón práctica. De esta manera, la razón práctica, por un lado, ordena realizar una 


\section{Martín Arias Albisu}

finalidad que coincide con algunos de los efectos de esos mecanismos naturales y, por el otro, condena la efectuación de los mismos mecanismos en el caso de que sean inmorales. Con respecto al último punto, debe señalarse que estos mecanismos no son necesariamente inmorales. Recordemos que, según ZeF, a fin de evitar la fusión de los diferentes Estados en una monarquía universal despótica y conquistadora, la garantía de la naturaleza introduce las diferencias de lengua y religión (véase II.2). Es evidente que esta diferenciación no es inmoral, aunque, en un primer momento, puede acarrear consecuencias inmorales ("la propensión al odio mutuo y al pretexto para la guerra", ZeF AA 08: 367. Traducción modificada).

Ocupémonos ahora de responder al segundo interrogante planteado, a saber, acerca del modo en que los mecanismos naturales se vinculan con un albedrío libre. Wood señala acertadamente que la identificación, por parte de Kant, de este mecanismo de la insociable sociabilidad depende de "consideraciones empíricas, a saber, una observación muy general" (Wood, 2009, 114). Mediante tal mecanismo se intentan explicar, conjeturalmente, ciertas tendencias en el accionar de la especie humana. Consideramos que la existencia de tales tendencias no exime a los individuos de su responsabilidad al decidir efectuar las acciones que vienen indicadas o sugeridas por estos mecanismos. Si al dejarse llevar por estos mecanismos generadores de tendencias los individuos cometen acciones contrarias a la moralidad, ellos son enteramente culpables con respecto a las mismas. Efectivamente, como explica Kant en Contestación a la pregunta: ¿Qué es la ilustración? (WA, 1784), los seres humanos son culpables por no pensar por sí mismos y no oír a su propia conciencia moral (AA 08: 35).

Hemos visto que, a partir del antagonismo entre los individuos, mediante el cual se desarrollan las disposiciones humanas, puede conformarse una condición civil, que en sus inicios ha de ser imperfecta. La limitación de este antagonismo mediante semejante condición civil permite que se intensifique el desarrollo de las facultades humanas. De este modo, los seres humanos se aproximan a una época que Kant denomina de "Ilustración" (Aufklärung; WA, AA 08: 40). Gracias a esta llustración, cada vez más individuos piensan por sí mismos y se sirven de su conciencia moral. 
Kant y la teleología de la naturaleza: acerca de la intención de la naturaleza en Idea para una historia universal en intención cosmopolita y la garantía de la naturaleza en Hacia la paz perpetua

Lo anterior no implica que, para Kant, el antagonismo entre individuos y entre Estados no se encuentre vigente en la época de llustración en la que escribe. Sin embargo, debe destacarse que el filósofo de Königsberg considera que la humanidad se encuentra en una época en la que cada vez más individuos piensan, acerca de cuestiones teóricas y prácticas, de un modo autónomo. "Gracias al arte y la ciencia somos extraordinariamente cultos. Estamos civilizados hasta la exageración en lo que atañe a todo tipo de cortesía social y a los buenos modales. Pero para consideramos moralizados queda todavía mucho." (IaG, AA 08: 26). Este desarrollo de las facultades humanas, especialmente el que nos cuesta más esfuerzo, a saber, el de la razón práctica, hace posible que, por ejemplo, cada vez más individuos cooperen para mejorar los asuntos públicos, tanto internos como externos, de su Estado, el cual es muy imperfecto en sus orígenes. ${ }^{16}$ Sin embargo, este desarrollo no hace necesario que los individuos decidan colaborar con el mejoramiento del Estado, es decir, no determina necesariamente el albedrío de los individuos.

En pocas palabras, un mecanismo de la naturaleza como el que consiste en el antagonismo entre los individuos genera una tendencia en las acciones libres de los seres humanos que posibilita, mediante la instauración de una condición civil, el desarrollo cada vez más pleno de facultades humanas como la razón práctica. De esta manera se llega a una época de llustración, en la que cada vez más individuos se apropian de su razón práctica, esto es, reconocen enteramente su propia ley moral. En este punto, los ciudadanos de buena voluntad pueden contribuir al perfeccionamiento

\footnotetext{
${ }^{16}$ Véase la famosa doctrina kantiana del "uso público de la razón" en WA. "Por uso público de la propia razón entiendo aquél que cualquiera puede hacer, como alguien docto, ante todo ese público que configura el universo de los lectores. Denomino uso privado al que cabe hacer de la propia razón en una determinada función o puesto civil, que se le haya confiado." (WA, AA 08: 37). Por ejemplo, en el caso de un sacerdote, la diferencia entre los usos privado y público de la razón es la siguiente: "un sacerdote está obligado a hacer sus homilías, dirigidas a sus catecúmenos y feligreses, con arreglo al credo de aquella Iglesia a la que sirve; puesto que fue aceptado en ella bajo esa condición. Pero en cuanto persona docta tiene plena libertad, además de la vocación para hacerlo así, de participar al público todos sus bienintencionados y cuidadosamente revisados pensamientos sobre las deficiencias de aquel credo, así como sus propuestas tendentes a mejorar la implantación de la religión y la comunidad eclesiástica." (WA, AA 08: 38). El uso público de la razón, en conclusión, hace posible que los ciudadanos contribuyan a la reforma y mejora gradual del orden vigente. Por otro lado, para una enumeración de las tareas que, en consonancia con el planteo kantiano, pueden desempeñar los individuos para aproximarse al ideal de la paz perpetua, véase Caranti $(2014,159)$.
} 
del Estado. Pero sigue siendo el libre albedrío de los individuos el que dictaminará si ellos obrarán de esta manera o de otra.

\section{Conclusiones}

En laG Kant presenta el concepto de una intención de la naturaleza que permite discernir una finalidad en la historia humana. Tal intención de la naturaleza consiste en el desarrollo pleno, en general, de las disposiciones humanas y, en particular, de la disposición racional humana. Dado que estas disposiciones pueden realizarse integralmente sólo en el marco de una constitución civil justa, Kant considera que la tarea más importante de los seres humanos es la conformación de dicha constitución. Asimismo, como el establecimiento de esta constitución sería vano si existiese una situación de guerra entre los diferentes Estados, se hace necesario formar una federación de los mismos, dotada de un poder coactivo, que administre conforme a derecho los conflictos entre los Estados miembros.

La intención de la naturaleza de laG puede entenderse como una idea regulativa de la razón teórica. La fundamentación de la teleología contenida en esta idea se encuentra, consiguientemente, en la doctrina del uso regulativo de la razón teórica expuesto en KrV. Por otro lado, el mecanismo del que se sirve la naturaleza para aproximar al ser humano a la finalidad de la historia es el antagonismo entre los individuos y entre los Estados.

En ZeF, Kant introduce el concepto de una garantía de la naturaleza para la aproximación progresiva del ser humano a la idea de una paz perpetua. Esta condición de paz perpetua tiene la forma política de una confederación voluntaria de Estados sin constitución ni poder coactivo. La garantía de la naturaleza en cuestión no es de naturaleza teórica, sino práctica, porque su función no es suministrar conocimiento, sino proveer sustento a la finalidad moral de realizar asintóticamente la idea de la paz perpetua. Los mecanismos de los que se sirve la naturaleza para aproximar al ser humano a la paz perpetua son examinados por Kant en relación con las tres esferas del derecho público, a saber, el derecho político, el derecho de gentes y el derecho 
Kant y la teleología de la naturaleza: acerca de la intención de la naturaleza en Idea para una historia universal en intención cosmopolita y la garantía de la naturaleza en Hacia la paz perpetua

cosmopolita. Por último, notemos que la garantía de la naturaleza es fundamentada por la doctrina teleológica de la naturaleza presentada en KU.

\section{Bibliografía}

Allison, H. E. (2004). Kant's Transcendental Idealism. Revised and Enlarged Edition. New Haven/London: Yale University Press.

Allison, H. E. (2009). Teleology and history in Kant: the critical foundations of Kant's philosophy of history. En A. Oksenberg Rorty y J. Schmidt (comps.), Kant's Idea for a Universal History with a Cosmopolitan Aim. A Critical Guide (pp. 24-45). Cambridge: Cambridge University Press.

Ameriks, K. (2009). The purposive development of human capacities. En A. Oksenberg Rorty y J. Schmidt (comps.), Kant's Idea for a Universal History with a Cosmopolitan Aim. A Critical Guide (pp. 46-67). Cambridge: Cambridge University Press.

Caranti, L. (2014). The Guarantee of Perpetual Peace: Three Concerns. En P. Formosa, A. Goldman y T. Patrone (comps.), Politics and Teleology in Kant (pp. 145-162). Cardiff: University of Wales Press.

Fiegle, T. (2014). Teleology in Kant's Philosophy of History and Political Philosophy. En P. Formosa, A. Goldman y T. Patrone (comps.), Politics and Teleology in Kant (pp. 163-179). Cardiff: University of Wales Press.

Flikschuh, K. (2006). Reason and Nature: Kant's Teleological Argument in Perpetual Peace. En G. Bird (comp.), A Companion to Kant (pp. 383-396). Oxford/Malden: Blackwell Publishing.

Hoesch, M. (2015). Garantie. En M. Willaschek, J. Stolzenberg, G. Mohr y S. Bacin, (comps.), Kant-Lexikon (pp. 669-670). Berlin: De Gruyter.

Höffe, O. (1992). "Even a Nation of Devils Needs the State": the Dilemma of Natural Justice". En H. Williams (comp.), Essays on Kant's Political Philosophy (pp. 120-142). Cardiff: University of Wales Press. 


\section{Martín Arias Albisu}

Kant, I. (1900ss.). Kant's gesammelte Schriften. Edición de Deutsche (anteriormente Königlich Preußische) Akademie der Wissenschaften. Berlin y otros: Walter de Gruyter y predecesores [Akademie-Ausgabe = AA].

Kant, I. (1981). La religión dentro de los límites de la mera razón. (F. Martínez Marzoa, Trad.). Madrid: Alianza. [RGV].

Kant, I. (1996). Sobre la paz perpetua. (Joaquín Abellán, Trad.). Madrid: Tecnos. [ZeF]

Kant, I. (2003). Crítica del discernimiento. (R. R. Aramayo y S. Mas, Trad.). Mínimo Tránsito: Madrid. [KU].

Kant, I. (2009). Crítica de la razón pura. (M. Caimi, Trad.). México D. F.: Fondo de Cultura Económica. [KrV].

Kant, I. (2011). Crítica de la razón práctica. (D. M. Granja Castro, Trad.). México D. F.: Fondo de Cultura Económica. [KpV].

Kant, I. (2013a): Contestación a la pregunta: ¿Qué es la ilustración? (R. R. Aramayo, Trad.). En R. R. Aramayo (comp.), ¿Qué es la llustración? Y otros escritos de ética, política y filosofía de la historia (pp. 85-98). Madrid: Alianza. [WA].

Kant, I. (2013b): Idea para una historia universal en clave cosmopolita (R. R. Aramayo y C. Roldán Panadero, Trad.). En R. R. Aramayo (comp.), ¿Qué es la Ilustración? Y otros escritos de ética, política y filosofía de la historia (pp. 99125). Madrid: Alianza. [laG].

Kleingeld, P. (2004). Approaching Perpetual Peace: Kant's Defence of a League of States and his Ideal of a World Federation. European Journal of Philosophy, 12(3), 304-325. Doi: https://doi.org/10.1111/j.0966-8373.2004.00212.x

Kleingeld, P. (2008). Kant on historiography and the use of regulative ideas. Studies in History and Philosophy of Science, 39(4), 523-528. Doi: https://doi.org/10.1016/j.shpsa.2008.09.006

Kleingeld, P. (2009). Kant's changing cosmopolitanism. En A. Oksenberg Rorty y J. Schmidt (comps.), Kant's Idea for a Universal History with a Cosmopolitan Aim. A Critical Guide (pp. 171-186). Cambridge: Cambridge University Press. 
Kant y la teleología de la naturaleza: acerca de la intención de la naturaleza en Idea para una historia universal en intención cosmopolita y la garantía de la naturaleza en Hacia la paz perpetua

Louden, R. B. (2014). Cosmopolitical unity: the final destiny of the human species. En A. Cohen (comp.), Kant's Lectures on Anthropology. A Critical Guide (pp. 211-229). Cambridge: Cambridge University Press.

Shell, S. (2015). Anlage. En M. Willaschek, J. Stolzenberg, G. Mohr y S. Bacin (comps.), Kant-Lexikon (pp. 96-97). Berlin: De Gruyter.

Vaki, F. (2014). What Are We Allowed to Hope? Kant's Philosophy of History as Political Philosophy. En P. Formosa, A. Goldman y T. Patrone (comps.), Politics and Teleology in Kant (pp. 194-210). Cardiff: University of Wales Press.

Williams, H. (1983). Kant's Political Philosophy. Oxford: Basic Blackwell.

Wood, A. W. (2006). Kant's Philosophy of History. En P. Kleingeld (comp.), Immanuel Kant. Toward Perpetual Peace and Other Writings on Politics, Peace, and History (pp. 243-262). New Haven/London: Yale University Press.

Wood, A. W. (2009). Kant's Fourth Proposition: the unsociable sociability of human nature. En A. Oksenberg Rorty y J. Schmidt (comps.), Kant's Idea for a Universal History with a Cosmopolitan Aim. A Critical Guide (pp. 112-128). Cambridge: Cambridge University Press.

Zocher, R. (1958). Zu Kants transzendentaler Deduktion der Ideen der reinen Vernunft. Zeitschrift für philosophische Forschung, 12(1), 43-58. 